\title{
An approach for detecting deviations in daily routine for long-term behavior analysis
}

\author{
Denis Elbert \\ Hochschule Mannheim \\ Mannheim, Germany \\ denis.elbert@gmx.de
}

\author{
Holger Storf, Michael Eisenbarth, Özgür Ünalan, \\ Mario Schmitt \\ Fraunhofer IESE \\ Kaiserslautern, Germany \\ firstname.lastname@iese.fraunhofer.de
}

\begin{abstract}
Rendering and offering adequate reminder services in a situation-aware, proactive manner and providing information for diagnosis support is a major issue for Ambient Assisted Living systems when it comes to dealing with persons suffering from mild dementia. One great challenge therefore is to reliably recognize and assess the long-term behavior of assisted persons. In the context of diagnosis support for caregivers or practitioners, deviations in the daily routine of a person with mild dementia might be an indicator of a deterioration of the affected person's cognitive condition. Based on this information, adequate help can be provided. We developed an approach to processing information regarding the modeling of daily routines and a comparison to previous days. Our solution can be seen as a combination of three approaches: a cosinor analysis based on the theory of circadian rhythms as a special representative of regression analysis, a histogram-based approach based on movement data, and a probabilistic model of behavior (PMB) based on the person's activities of daily living (ADL).
\end{abstract} Living

Behavior Monitoring; Circadian Rhythm, Ambient Assisted

\section{INTRODUCTION}

Demographical, structural, and social trends are driven by an increasing number of elderly people and single households in many industrialized countries. The consequences are that more and more elderly people are forced to live alone, leading to a high number of emergencies reported late or remaining undiscovered. This fact has dramatic effects on public and private health care, emergency medical services, and the individuals themselves. Due to this increase in emergency situations, health care costs in the coming years will rise drastically. In addition to the growing number of critical emergency situations, the number of people suffering from typical diseases of the older generation, such as dementia, will rise significantly. In last year's World Alzheimer Report, Alzheimer's Disease International estimated that there are 35.6 million people living with dementia worldwide in 2010; these numbers will increase to 65.7 million by 2030 and to 115.4 million by 2050 [1].

In the EU-funded project CCE (Connected Care for Elderly Persons Suffering from Dementia) [2], a system concept for supporting people suffering from dementia in their daily lives will be developed, implemented as a prototype, and validated.

People with dementia generally suffer from "gradual memory loss, decreasing ability to perform routine daily living tasks, impaired judgment, disorientation, personality changes, loss of language skills, and behavior disturbance" [3]. As the disease deteriorates, dementia sufferers find it more and more difficult to "comprehend language in written and spoken form" [4]. Short-term memory problems create further difficulties, as people become unable to follow a conversation, forgetting what was said just a little while ago. Furthermore, given that they become easily disoriented and distracted, it makes it very difficult to communicate when there are many people all talking at the same time.

The focus of CCE lies on providing assistance to people with cognitive impairments by giving them reminders regarding specific appointments or helping them interact with everyday devices like the door key before leaving the house in order to prevent unpleasant situations. The reminders for appointments such as a visit to the doctor or the visit of a family member or neighbor should be seen as memory support. Besides these special appointments, people with dementia are also reminded to perform normal daily activities, the so-called ADLs [5], in order to follow their daily routines.

As a control mechanism, a software component - the circadian rhythm score (CRS) calculator - for analyzing a person's daily routine has been developed. On the one hand, the goal of this component is to detect deteriorations in the person's long-term behavior; on the other hand, the interpretation of the daily rhythm should be an indicator of how successful the reminder services might be.

Our system monitors the behavior patterns of the assisted person through unobtrusive sensors embedded into their home living environment. It utilizes the sensor data to extract typical behavior patterns. To this end, it uses a model that represents typical or acceptable behavior patterns based on the ADLs and other defined activities. The aggregated activity information is provided to the CRS Calculator. The daily calculated score is used by the Trend Analyzer for detecting tendencies.

The goal of this paper is to: (i) provide insights into the system, (ii) show that our approach to CRS calculation is suitable for the successful interpretation of daily routines, and (iii) report on our experiences during the development.

The remainder of this paper is structured as follows: In chapter 2, we briefly describe the ambient assisted living domain and the degree to which the CCE project is related to this area of research. Chapter 3 provides details about the CCE 
system into which the CRS calculator component will be integrated. In chapter 4, we introduce several methods of behavior monitoring known from the literature that we utilized to implement the CRS calculator. Details of our final approach to calculating the CRS are presented in chapter 5. Finally, we draw conclusions from our approach and provide an outlook on future work.

\section{AMBIENT Assisted Living AND The CCE PROJECT}

Ambient Assisted Living (AAL) [6] denotes concepts, products, and services that interlink and improve new technologies and social systems with the aim of enhancing the quality of life for all people during all stages of their lives. AAL can therefore be translated best as intelligent systems of assistance for a better and safer life [7]. The potential range of services belonging to the AAL domain is huge. It encompasses any assistive service that facilitates daily life.

In the context of automated emergency treatment, for instance, short-term situations comprise the automatic, reliable, and immediate detection of emergency situations such as sudden falls or helplessness, or long-term deviations from typical behavior and from vital parameters. Other types of critical situations from the medical point of view are special incidences of long-term trends that are indicators of mental changes, e.g., dementia, depression, or other age-related diseases. Of special interest in behavioral monitoring and health state assessment are the so-called activities of daily living (ADLs), which were defined in the Katz index [5] for the first time. Based on ADLs, long-term trend analyses can be performed and checked against the assisted person's normal behavior and additional clinical information. Thus, deviations or inconsistencies provide indicators of the assisted person's health status and proactive assistance can be initialized if needed. This is particularly useful in case of dementia. Based on the recognized situation and health condition, adequate assistance services can be rendered in a proactive and preventive way [8]. These services are one of the main objectives of the CCE project, which focuses on providing assistance based on ICT technologies, especially for elderly persons suffering from dementia.

\section{SYSTEM INTEGRATION}

In order to detect long-term deviations from the typical behavior of the assisted person, several subcomponents have been introduced that provide specialized information about the chronic behavior of the person. For example, the sleep monitor subcomponent aggregates information about the sleep behavior, whereas the activity score calculator characterizes the person's physical activity. All subcomponents together form the so-called long-term behavior monitoring component (LBM) of the system. The trend analyzer (see Fig. 1) is part of the LBM and detects critical changes in the behavior of the assisted person based on the aggregated information from the different LBM subcomponents.

The functionality of the reminder service mentioned above has been implemented within the short-term behavior monitoring component (SBM). Both the LBM and the SBM require information about the ADLs of the assisted person. This information is obtained from the so-called activity recognizer component (AR). The AR processes the sensor events received from the AAL environment and combines different approaches known from artificial intelligence, such as case-based reasoning, rule-based reasoning, time maps, and fuzzy approaches, to detect the ADLs [9]. Once an ADL is detected, an associated event is created and stored in a database (DB). Other components such as LBM or SBM can access the information by either registering for the particular ADL event (asynchronous communication) or by querying the events from the DB (synchronous connection). In both cases, the communication is realized by an information broker (IB). In other words, the entire communication between all components is established by the IB. Fig. 1 gives an overview of the system architecture described above.

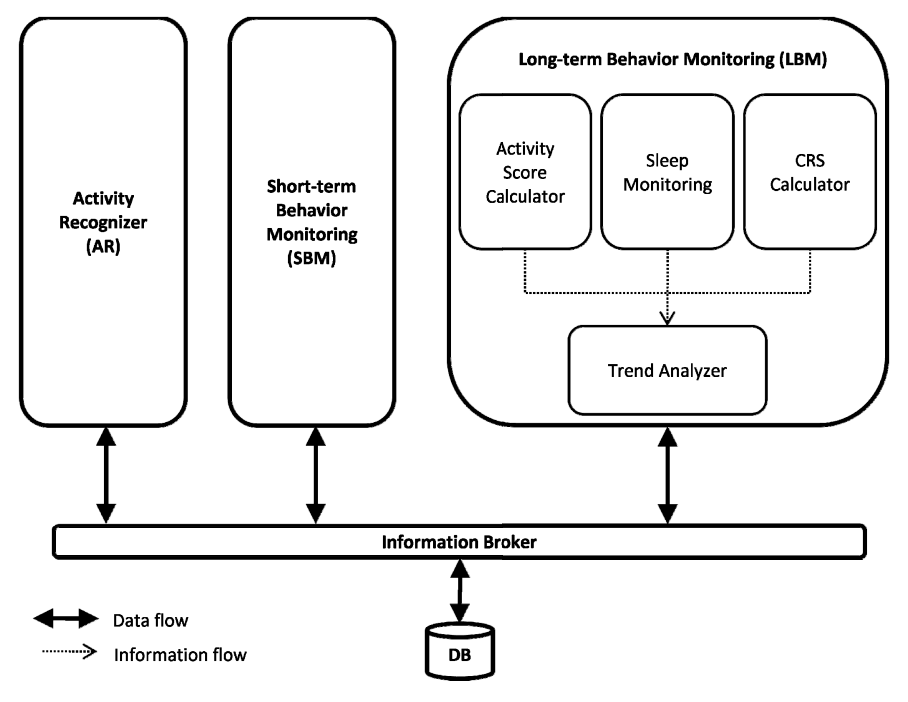

Figure 1. CCE system design for behavior monitoring.

This paper focuses on one of the LBM subcomponents called the circadian rhythm score (CRS) calculator. The CRS represents the deviation from the assisted person's daily activity or daily routine. Over a 24-hour period, and for each day of the week, it provides information about how similar the daily routine of the current day (the current behavior) has been in comparison to the "normal" or "typical" behavior. With "normal" we mean the average behavior that has been observed over a longer period of time. The final resulting score calculated by the CRS subcomponent has been designed to take values ranging from 0 to 1 . The closer the value is to 1 , the less the current behavior deviates from the "normal" behavior, indicating a strong daily routine. In contrast, smaller values (approaching 0) represent a larger deviation from the typical behavior, indicating a weak daily routine.

As mentioned above, the CRS is calculated for each day of the week, with the individual scores being stored in the DB for the purpose of being processed further by the trend analyzer component. The trend analyzer then analyzes the scores with respect to critical changes in the assisted person's daily routine.

In summary, the main challenge of the CRS calculator component is (i) to build a model that represents the typical behavior of the assisted person and (ii) to apply this model in order to provide information about the deviation from the usual behavior for each day of the week. The following chapters explain our approach to the implementation of the presented CRS calculator component, which comprises the detection of 
changes in the typical behavior of an assisted person using AAL technology.

\section{Modeling Daily Routines - Problems AND APPROACHES}

Regarding our problem of detecting deviations in a person's long-term behavior, the literature provides dozens of different approaches. Some are based on general mathematical principles such as regression or time series analysis; others evolved in studies, where the influence of dementia and Alzheimer's disease on the lifestyle of affected people was investigated. Below, we introduce a selected subset of these approaches that are also feasible for our purposes.

The first approach is based on the theory of so-called circadian rhythms (CRs) from which our CRS calculator component takes its name.

CRs play an important role in the field of chronobiology and represent endogenous periodic rhythms that have a period of approximately 24 hours. As a result of our rhythmic environment, every human possesses an "internal clock", which follows such CRs [10]. This internal clock controls functions such as body temperature, rhythms in hormones, and - most important in this context - the rest-activity cycle [11]. Since dementia has been shown to have a negative influence on the functionality of the patient's internal clock, it consequently affects his rest-activity cycle, which results in disturbed sleepwake patterns and therefore in changes in the person's daily behavior [11][12].

During years of research in the area of CRs, especially while examining the relationship between dementia and CRs, a method called actigraphy has been used to study the restactivity pattern of human subjects (often elderly people with and without dementia). Actigraphy uses small devices named actigraphs that are worn on the wrist and record movement data through their built-in accelerometer. Several studies have demonstrated that recordings of actigraphic data show a circadian pattern. Also, changes in the CR were revealed when data had been recorded continuously over a longer period of time (weeks, months and years) [13].

Fig. 2 gives an example of actigraphic data, which was collected and plotted over 14 consecutive days. The data originates from two different subjects: a healthy person (upper half) and a person suffering from dementia (lower half). Each row plots the accumulated movement data (vertical axis) over a time period of 48 hours (horizontal axis).

The graphic shows a clear periodic pattern within 24 hours in case of the healthy subject with high activity during day time and very low activity during night time. The second graph however, reveals strongly disturbed sleep phases indicated by almost constant diurnal and nocturnal activity.

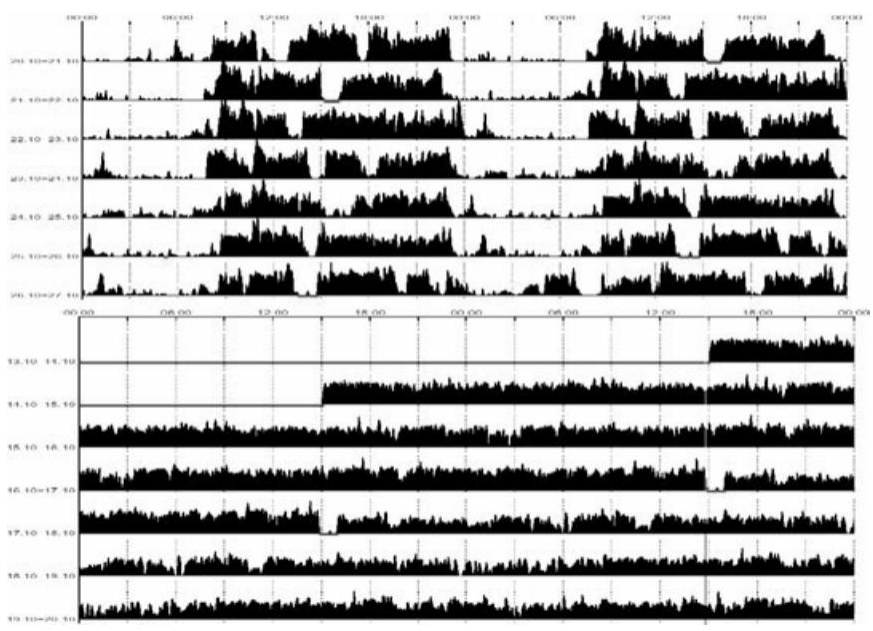

Figure 2. Plots of actigraph data for a healthy person and a person suffering from dementia [14].

In regard to our CRS calculator component, these results from actigraphy could be utilized to build a model of the typical behavior of an assisted person. The most popular method for building such a model has been cosinor analysis, a specialized kind of regression analysis, in which “... a cosine curve with a period at or near 24 hours is fit to the data by the least-squares method" [13]. As can be seen in Fig. 3, the fitted curve is characterized by three parameters: The acrophase defines the point of time when the maximum activity is recorded, the amplitude is the difference between maximum and minimum activity, and the mesor specifies the mean activity [13].

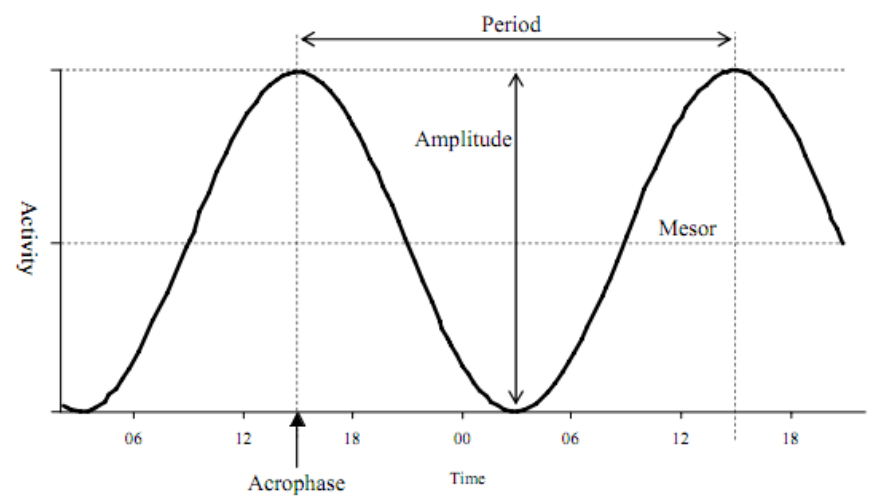

Figure 3. Circadian Rhythm representation used by Cosinor analysis [13].

Applied to the given example shown in Fig. 2, cosinor analysis fits the curve in such a way that the wave crest is assigned to the occurrence of high activity during daytime, whereas the wave trough appears in the area of low activity during nighttime. In this example, the models created for (i) the healthy subject and (ii) the subject suffering from dementia differ most notably in terms of amplitude and mesor: (i) has greater amplitude compared to almost zero amplitude in (ii), whereas the mesor is higher in (ii) than in (i).

Because actigraphic data usually deviates from the exact shape of a cosine curve, an extension to the traditional cosinor analysis has been developed, in which a total of five parameters describe the fitted cosine model. Additional parameters such as 
$\gamma$ - the steepness of the rise and fall of the curve - ensure a better fit to the actual actigraphic data. Ancoli et al. have set a good example for the application of extended cosine analysis during their studies of CRs [15].

In order to model typical behavior as defined by our CRS, our suggestion is to build the (extended) cosinor model for each day of a predefined training period and use the average as the representation of normal behavior $\left(C M_{\text {norm }}\right)$. Of course, not all recorded data might be representative in terms of long-term behavior analysis (e.g., due to illnesses), so some of the data may have to be ignored or omitted, but this issue is beyond the focus of this paper. If the cosinor model is calculated for a single day $\left(C M_{\text {curr }}\right)$, the deviation of this day from normal behavior is represented by the difference of $C M_{\text {norm }}$ and $C M_{\text {curr }}$.

In summary, cosine analysis in conjunction with actigraphy is a well-known method for extracting models of the sleepwake patterns - as well as the daily behavior - of an assisted person and can be used to calculate the CRS as suggested above. As a disadvantage, it requires the person to wear a small actigraph device. Because our AAL environment is aimed at unobtrusive ways of measurement, the presented solution can only be considered to be an additional feature in the CRS calculation and must be combined with another approach based on ADLs.

Besides this cosinor analysis approach based on CRs, our problem could also be tackled by another broad area of research called Activity Monitoring, which focuses on the automatic detection of abnormal behavior. In this context, behavior is not exclusively related to a person's behavior, but to the behavior of a large population of entities in general. For example, Activity Monitoring also includes computer intrusion detection or some forms of fault detection (e.g., fault detection in engine turbines [16]) and therefore provides solutions for a huge class of problems [17].

Activity Monitoring distinguishes between two basic methods: A profiling method builds a model of normal behavior and allows for detecting deviations from this model, whereas a discriminating method builds a model of both normal and abnormal behavior. The latter assumes that abnormal behavior has already occurred in the training data set and thereby learns to discriminate normal from abnormal behavior [17].

For both methods, another distinction between uniform and individual profiling/discriminating methods (see Fig. 4) can be made [17][18]. The uniform approach builds only one model for the entire population of entities, assuming that the behavior is uniform across the different entities. In contrast, the individual approach creates a model for each single entity.

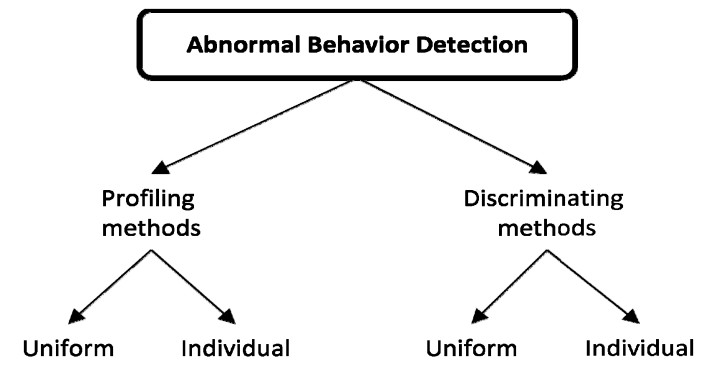

Figure 4. Classification of activity monitoring approaches [18].
In relation to our problem of finding deviations in the behavior of an assisted person within an AAL environment, only profiling methods are applicable. This is due to the fact that - in the context of our AAL environment - countless forms of abnormal behavior are imaginable. This makes it impossible to build a model of abnormality. Furthermore, our CRS calculator component aims at finding deviations from the personal daily routine of the assisted person. This requires an individual approach. In summary, we have concluded that an individual profiling approach is needed for calculating the CRS.

Virone et al. [19] presented a so-called histogram-based approach, which falls into the category of individual profiling. In this approach, they used data from an in-home monitoring system (IMS) equipped with motion sensors placed in every room of the monitored environment. Based on this data, they measured the amount of time spent in each room for each hour of the day (referred to as presence-based data) as well as the activity level during the time of presence given by the average number of motion events per room (referred to as activitylevel-based data). Normal behavior was modeled by collecting data from a multiple-day training period and by calculating mean values $m$ and standard deviations $s$ for both the presencebased and the activity-level-based data.

They utilized the extracted model of normal behavior in a tool called SAMCAD (Software for Automatic Measurement of Circadian Activity Deviation), which generates alerts whenever abnormal behavior is detected. Here, behavior is considered as being abnormal if it is outside an interval $\left[\mathrm{S}_{1} *\right.$, $\mathrm{S}_{1}$ ] or $\left[\mathrm{S}_{2}{ }^{*}, \mathrm{~S}_{2}\right]$, where behavior outside the first interval is called non-habitual and behavior outside the second interval alarmingly non-habitual (see Fig. 5). The intervals were defined as

$$
\begin{gathered}
{\left[\mathrm{S}_{1} *, \mathrm{~S}_{1}\right]=\left[m-\mu 1 \cdot s, m+\mu_{1} \cdot s\right] \text { and }} \\
{\left[\mathrm{S}_{2}{ }^{*}, \mathrm{~S}_{2}\right]=\left[m-\mu_{2} \cdot \mathrm{s}, m+\mu_{2} \cdot s\right]}
\end{gathered}
$$

where the parameters $\mu_{1}$ and $\mu_{2}$ set the thresholds for the two different types of alerts (non-habitual and alarmingly nonhabitual) triggered by the SAMCAD application.

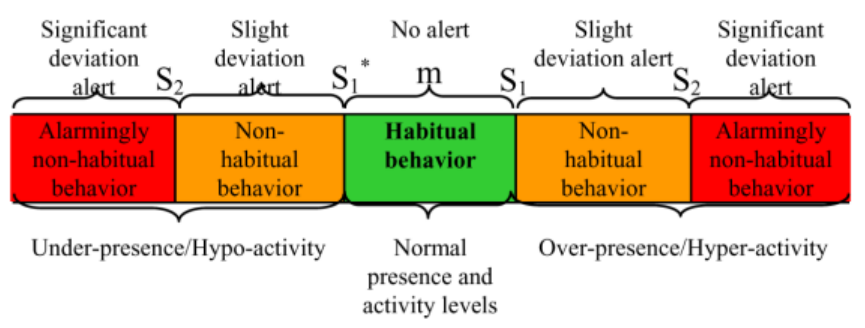

Figure 5. Illustration of different alert types defined by Virone et al. [19].

Ohta et al. [20] followed a similar approach as the one described above, but added the presence-based and activitylevel-based values up for each day and not for each hour. They also introduced other variables to be measured, such as the daily distance moved by a person [18].

While Virone et al. and Ohta et al. used the histogrambased approach to generate alerts on abnormal behavior that 
exceeds the thresholds, our idea is to assume a Gaussian distribution based on the aforementioned mean value $m$ and standard deviation $s$ and to compute the likelihood of a new behavior occurring. More details about this can be found in chapter 5 .

Another method that can be used for individual profiling is to build a so-called probabilistic model of behavior (PMB). This idea has been presented in [18] and relies on the assumption that a person's daily activities (ADLs) follow regular patterns, which can be learned by a probabilistic model. For example, it is assumed that a healthy person who is not suffering from dementia gets up at approximately the same time in the mornings or sleeps approximately the same amount of time every night. Case study experiments have supported these assumptions [18].

To build the model, each ADL (e.g., 'sleep') is associated with a set of $I$ attributes $a_{i}$. The attributes can be expressed in an I-dimensional vector $\vec{x}$, with $\vec{x}$ representing the occurrence of one activity. In the sleep activity example, attributes may be:

- Sleep onset time

- Sleep duration

- Number of (short) sleep interruptions

Depending on what kind of probabilistic function is used, the model is represented by a parameter set $\lambda$. In case of a Gaussian density function, the parameter set $\lambda$ would be composed of the expected value and the variance. These parameters are trained or estimated by using multiple occurrences of activities $\vec{x}_{n}$ as a training set. As a result, the log likelihood $\Lambda$ of a behavior or activity occurrence $\vec{x}$ being normal can be defined as

$$
\Lambda(\vec{x})=\log (P(\vec{x} \mid \lambda)) .
$$

Similar to the approach taken in the histogram-based approach by Virone et al., the authors in [18] defined a threshold $\tau$ to differentiate between normal and abnormal behavior. They considered behavior as being abnormal if $\Lambda(\vec{x}) \leq \tau$.

In our approach to calculating the CRS, we made use of the basic concept of PMB and implemented this method as the core element of our CRS calculator component, which is explained in the following chapter.

\section{FINAL APPROACH}

In the previous chapter, we introduced three different methods that may be useful for the implementation of our CRS component: cosinor analysis based on the theory of circadian rhythms as a special representative of regression analysis, a histogram-based approach based on movement data, and PMB based on a person's ADLs.

Our strategy is to combine these (three) approaches, with each method contributing a so-called sub-score to the final CRS (see Fig. 6). Weights associated with each method allow us to control and align the influence of each sub-score on the CRS. It is therefore possible to add methods to the CRS calculation or even to exclude them, if necessary. In our current implementation, for example, the cosinor analysis method has been omitted because our AAL environment does not provide actigraph-related data (see previous chapter). But the proposed system design will allow us to include it in the CRS calculation in the future if actigraph data becomes available.

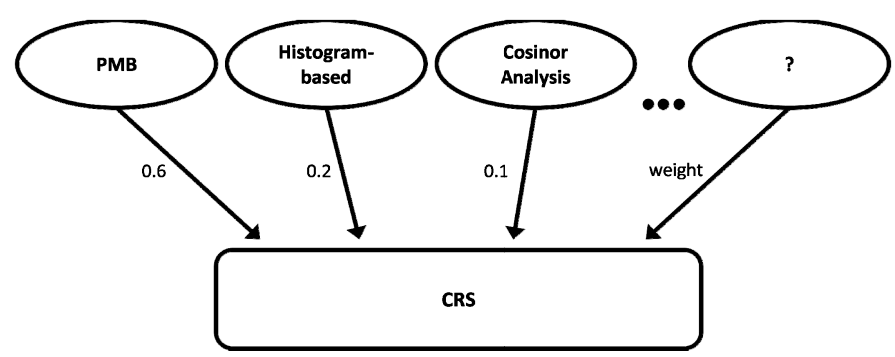

Figure 6. Multiple sub-components building the final CRS in the CRS calculator component.

In the following, we explain our realization of the PMBand histogram-based approach. Here, the PMB approach was selected to be the core element of our CRS calculation because studies conducted in [18] showed promising results.

As stated above, PMB requires a probabilistic density function that serves as a model for normal behavior. Of course, the exact shape of the density function is not known, because we do not have knowledge about what the data we want to model, i.e., the activity patterns, will look like. But the literature on statistical pattern recognition offers methods for estimating the probabilistic density function. One of the most popular ones is the Gaussian mixture model (GMM) [16], which we used for our purposes.

A GMM is a linear combination of $K$ independent multivariate Gaussian kernels $\mathcal{N}\left(\vec{x} \mid \mu_{k}, \Sigma_{k}\right)$, each of them specified by its mean value $\mu_{k}$ and variance $\Sigma_{k}$. $\omega_{k}$ is the weight of Gaussian k. All weights must sum up to 1 to preserve the characteristics of a probabilistic density function. The dimension of the Gaussians equals the number of attributes Ithat have been associated with an activity (see chapter 4 ). For a single activity or behavior $\vec{x}$, the likelihood is given by

$$
\begin{gathered}
P(\vec{x} \mid \lambda)=\sum_{k}^{K} \omega_{k} \cdot \mathcal{N}\left(\vec{x} \mid \mu_{k}, \Sigma_{k}\right) \text { with } \\
\lambda=\left\{\omega_{k}, \mu_{k}, \Sigma_{k}\right\}_{k=1}^{K} .
\end{gathered}
$$

As can be seen in (5), the GMM is specified by a total of $3 * \mathrm{~K}$ parameters. Once these parameters are known or learned using a training data set, the likelihood of a single behavior or activity occurring can be calculated. The likelihood serves as a measure for the deviation from normal behavior (represented by the GMM) or as a measure of abnormality, respectively. The smaller the likelihood $P(\vec{x} \mid \lambda)$, the more the current observed behavior $\vec{x}$ deviates from normal behavior represented by $\lambda$.

Given a series of $\mathrm{N}$ activities $\vec{x}_{n}$ (the training data set), it is possible to estimate the parameters of $\lambda$ using different approaches such as the moments method, the moment generation function, graphical methods, Bayesian methods, and the maximum likelihood method [21]. We decided to follow the maximum likelihood approach. 
The goal of the maximum likelihood method is to find those parameters of the model for which the (log) likelihood of observing the activities $\vec{x}_{n}$ becomes maximized [21]. More formally, we are looking for $\lambda$, for which

$$
\begin{gathered}
\log \left[P\left(\lambda \mid \vec{x}_{1}, \ldots, \vec{x}_{n}\right)\right] \rightarrow \max \text { with } \\
P\left(\lambda \mid \vec{x}_{1}, \ldots, \vec{x}_{n}\right)=\prod_{n=1}^{N} P\left(\vec{x}_{n} \mid \lambda\right)
\end{gathered}
$$

Based on this principle, either an iterative or a recursive approach can be taken to finally estimate the parameters. The iterative approach usually produces better results, while the recursive approach is faster than the iterative one [21]. Since the detection of long-term deviations is not time-critical, we chose the iterative approach.

The most popular iterative method is the so-called Expectation-Maximization (EM) algorithm. Based on initial values for $\lambda$, the algorithm first computes the likelihood of the current estimate and uses the result to determine the new parameters that maximize the expectation [21].

One disadvantage of the EM algorithm is that we have to give an initial estimate for the GMM parameter set $\lambda$. The initial values will have a significant influence on the quality of the results of the EM algorithm [21] and must therefore be chosen with care. Also, the number of Gaussians $(K)$ must be known before. We addressed this issue by applying a special clustering technique known as DBSCAN (Density Based Spatial Clustering for Applications with Noise) [22] to the training data set. In contrast to the K-means algorithm, which is widely used to estimate the GMM parameters [21], no information about the number of clusters is needed in advance thanks to the density-based approach; DBSCAN will generate the clusters by itself and assign each data point $\vec{x}_{n}$ to one of the clusters. For each cluster - which can also be considered to represent one Gaussian kernel as part of the GMM - we calculated the mean value and variance that served as estimates for $\mu_{k}$ and $\Sigma_{k}$ respectively.

The DBSCAN algorithm relies on two parameters: minPts and $\varepsilon$. minPts is the minimum number of data points that are necessary to build a new cluster and $\varepsilon$ denotes the maximum distance between adjacent data points within one cluster. With respect to our set of activities, $\varepsilon$ therefore represents the maximum time difference between two activities for which these activities are considered to be the same kind of activity occurring at different dates. For example, if a person prepares lunch at 12 p.m. on Monday and a meal preparation is observed at $1.00 \mathrm{p} . \mathrm{m}$. on Tuesday, then the second activity is assumed to be lunch as well if $\varepsilon>60$ minutes. In case of $\varepsilon$ being equal to 20 minutes, the second activity would be assumed to be an additional meal preparation (and not the preparation of lunch).

In order to estimate both minPts and $\varepsilon$, we extended the histogram-based approach introduced in the previous chapter. In addition to the acquisition of presence-based and activitylevel-based data, we also record - for each type of activity as defined by the PMB approach - how often this activity occurs during one 24-hour period. For example, for the meal preparation activity, we count how often the person prepares meals during the course of one day. If this is done for each day of the training period, a histogram like the one shown in Fig. 7 can be derived from the records. In this diagram, the training period is 40 days. On twelve different days, food was prepared four times a day, whereas on only one day, food was prepared seven times. Based on the histogram data, the mean value and the standard deviation can be calculated, which leads to a Gaussian distribution as indicated by the red curve.

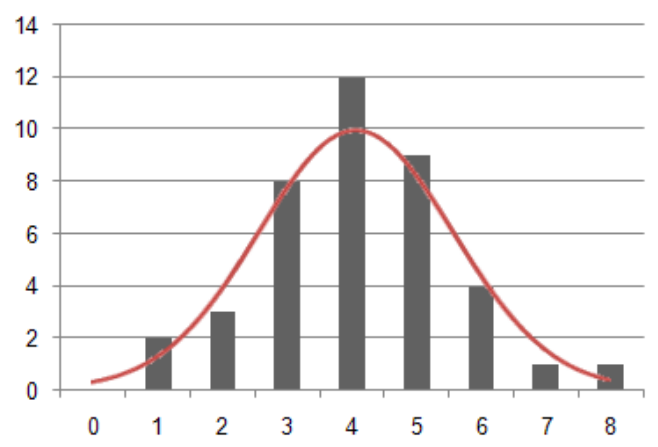

Figure 7. Histogram for the meal preparation activity based on records from a 40-day training period. The horizontal axis holds the number of occurences of the activity per day $\left(\mathrm{O}_{\mathrm{d}}\right)$, while the vertical axis tells how many days showed the respective $\mathrm{O}_{\mathrm{d}}$. The curve represents the Gaussian density function fitted to the histogram data.

We interpret the mean value as the expected occurrence frequency of an activity during a 24-hour period. In this example, we would therefore expect the person to prepare food four times during the day. This information can then be utilized to estimate the $\varepsilon$ parameter. One approach might be to simply divide 24 by the mean value; however, finding the most appropriate formula is part of ongoing work by the authors. As for minPts, we set this parameter to a percentage $\rho$ of the training period. For example, if $\rho$ is chosen to be $80 \%$ and the training period is 40 days, an activity must occur at least 32 times within the training period to be considered a regular activity. Conversely, if an activity occurs less than 9 times, it is considered to be exceptional behavior and is thus treated as an outlier in the focus of DBSCAN.

In summary, our final PMB approach for calculating the CRS sub-score is characterized by the following sequence of steps: We first define different kinds of activities (such as sleep, meal preparation, toilet usage, etc.) that have a set of attributes (e.g., start time, duration, etc.). For each of these activities, we build a GMM using activity data from within a predefined training period. The GMMs represent the behavioral model or the normal behavior for each activity. We then utilize the likelihood functions of the GMMs to calculate the probability of the current corresponding activity occurring and interpret that value as a measure for the deviation from normal behavior (score). We average multiple values for the same kind of activity (e.g., meal preparation) and get a final score for each type of activity. Since some types of activities may be more important than others, we assign each type of activity a weight and calculate the final CRS sub-score by combining all activity scores using the weights. Fig. 8 gives an overview of this procedure. 


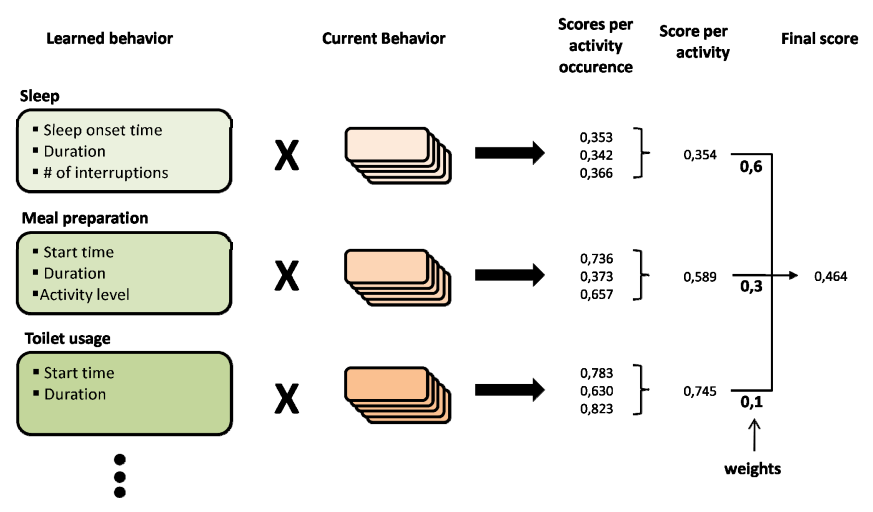

Figure 8. General approach for caluclating the CRS.

We did not only utilize the histogram-based approach to estimate the DBSCAN parameters, but also to establish a separate CRS calculation method as described in the beginning of this chapter. For that purpose, we counted the number of occurrences of each activity type during the current 24-hour period and used the Gaussian density function derived from the historical histogram (see Fig. 7) to calculate the likelihood of that observation. Again, the likelihood serves as a measure for abnormality. Similar to the PMB approach, we assigned each activity type a different weight and combined all values into a final sub-score.

As mentioned earlier, we decided to follow an individual profiling approach for calculating the CRS. This required building a model of normal behavior based on a training data set. As a consequence, we had to specify which data serve as training instances when calculating the daily CRS. For this purpose, we defined a configurable training interval $\left[T_{\text {start }}, T_{\text {end }}\right]$ in which $T_{\text {start }}$ denotes the first calendar date that will hold training data and $T_{\text {end }}$ denotes the last calendar date, respectively. Another parameter $\delta$ represents the distance from the current date to the training interval (or $T_{\text {end }}$ more precisely) measured in number of days (see Fig. 9). We identified three possibilities for the training interval to be configured:

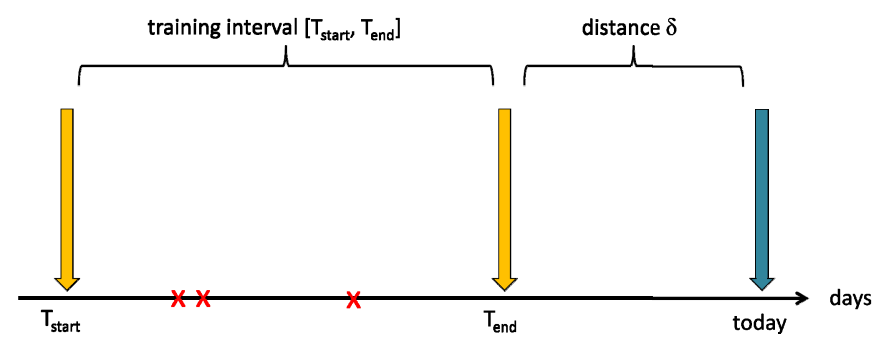

Figure 9. Definition of the training interval used for learning normal behavior in the CRS calculator component.

1. $T_{\text {start }}$ and $T_{\text {end }}$ are fixed. In this case, the training interval remains the same while the parameter $\delta$ is growing. As a consequence, the same model will be used for each CRS calculation (i.e., for consecutive days).

2. Only the parameter $\delta$ is fixed. In this case, the training interval shifts one day with each CRS calculation, thus representing a so-called sliding-window.

3. The parameter $\delta$ is fixed as well as $T_{\text {start }}$. In this case, the training interval grows by one day with each CRS calculation.
Ongoing evaluations of our implemented system will show which configuration for the training interval will produce the best results for the CRS.

Another problem is how to treat certain days within the training interval that are known to show irregular behavior (e.g., due to visits of relatives, illness, vacation, etc.) and are therefore not suitable for building a model of normality. The behavior might also show different patterns on weekends than during weekdays, which would require building separate models. The first issue is addressed by specifying fixed dates that the system will ignore when building the model (indicated by red crosses in Fig. 9).To solve the second problem, we defined groups of weekdays. For each group (e.g., \{Saturday, Sunday\}), a separate model was built using only data from the specified weekdays within the training interval. Afterwards, the appropriate model was used to calculate the CRS. For example, if the current day is Saturday, the model built from the group \{Saturday, Sunday\} was used to perform the CRS calculation. If the current day of the week is contained in more than one group, then the average was built from the CRS calculations of each group.

\section{CONCLUSION AND FUTURE WORK}

In this paper, we described our approach for recognizing deviations in the typical or normal behavior of elderly people suffering from dementia within an ambient assisted living environment (AAL). The goal was to develop a system component that calculates the so-called circadian rhythm score (CRS) based on the person's activities of daily living (ADLs). Here, the CRS represents the total degree of deviation indicated by a single real value ranging from 0 to 1 .

Several methods for behavior monitoring such as cosinor analysis, histogram-based approaches, and probabilistic models of behavior (PMB) known from the literature were introduced and combined into a final approach to calculate the CRS, with PMB being chosen as the core element of the calculation algorithm.

In the future, we plan to test and evaluate our approach in our own AAL testing environment as well as in real-life scenarios. During the course of these studies, we will also determine which system configuration settings will produce the best results for the CRS. These include the different weights of the CRS sub-scores, the weights for the activities defined as part of the PMB method, and the best setting for the training interval used to train our model of normal behavior.

\section{ACKNOWLEDGMENT}

Part of this work has been funded by the European Commission under project ref. aal-2008-1-101 CCE [2].

\section{REFERENCES}

[1] Alzheimer's Disease International (ADI), World Alzheimer Report 2010 - The Global Economic Impact of Dementia, 2010, pp. 4.

[2] CCE, Connected Care for Elderly Persons Suffering from Dementia, EU project, http:// www.cceproject.eu, last visited 20.02.2011.

[3] S. Burgener and P. Twigg,"Relationships among caregiver factors and quality of life in care recipients with irreversible dementia"Alzheimer Disease and Associated Disorders, Vol. 16, No. 2, pp. 88-102, 2002.

[4] N. J. Haak, "Maintaining connections: Understanding communication from the perspective of persons with dementia" Alzheimer's Care Quarterly, Vol. 3, No. 2, S. 122, 2002. 
[5] S. Katz, A.B. Ford, R.W. Moskowitz, B.A. Jackson, and M.W. Jaffe, "Studies of Illness in the Aged: The Index of ADL: A Standardized Measure of Biological and Psychosocial Function", Journal of the American Medical Association, 185(12), 1963, pp. 914 - 919.

[6] Ambient Assisted Living Joint Program, http://www.aal-europe.eu, last visited 01.03.2011.

[7] mst news: "Ambient Assisted Living", mstnews 06/07, http://www.mstnews.de/past-issues, last visited 01.03.2011.

[8] M. Nick and M. Becker, "A Hybrid Approach to Intelligent Living Assistance", Hybrid Intelligent Systems, Kaiserslautern, pp. 2-3, 2007.

[9] H. Storf, and M. Becker, "A Multi-Agent-based Activity Recognition Approach for Ambient Assisted Living", 3rd Workshop Artificial Intelligence Techniques for Ambient Intelligence, European Conference on Artificial Intelligence, Patras, Greece 2008.

[10] P.H. Redfern, J.M. Waterhouse and D.S. Minors, "Circadian Rhythms: Principles and Measurement”, Pharmac. Ther. Vol. 49, pp. 311-327, 1991.

[11] E. Werth, E. Savaskan, V. Knoblauch, P. Fontana Gasio, E. J. W. Van Someren, C. Hock, and A. Wirz-Justice, "Decline in Long-Term Circadian Rest-Activity Cycle Organization in a Patient with Dementia", Journal of Geriatric Psychiatry and Neurology, 2002.

[12] S. K. Roepke and S. Ancoli-Israel, "Sleep disorder in the elderly", Indian J Med Res 131, pp. 302-310, 2010.

[13] S. Ancoli-Israel, R. Cole, C. Alessi, M. Chambers, W. Moorcroft, C. P. Pollak, "The Role of Actigraphy in the Study of Sleep and Circadian Rhythms", American Academy of Sleep Medicine Review Paper, SLEEP 2003; 26(3) pp. 342-392, 2003.

[14] P. Paavilainen, I. Korhonen, J. Lötjönen, L. Cluitmans, M. Jylhä, A. Särelä, and M. Partinen, "Circadian activity rhythm in demented and non-demented nursing-home residents measured by telemetric actigraphy", 2004.

[15] J. Martin, M. Marler, T. Shochat, and S. Ancoli-Israel, „Circadian Rhythms of Agitation in Institutionalized Patients with Alzheimer's Disease“, Chronobiology International, 17(3), pp. 405-418, 2000.

[16] L. Tarassenko, A. Nairac, N. Townsend, I. Buxton, and P. Cowley, "Novelty detection for the identification of abnormalities", International Journal of Systems Science, 31:11, pp. 1427-1439, 2000.

[17] T. Fawcett, F. Provost, "Activity Monitoring: Noticing interesting changes in behavior", The Conference on Knowledge Discovery in Data, pp.53-62, 1999.

[18] F. Cardinaux, S. Brownsell, M. Hawley, D. Bradley, "Modelling of Behavioural Patterns for Abnormality Detection in the Context of Lifestyle Reassurance", CIARP 2008, LNCS 5197, pp. 243-251, 2008.

[19] G.Virone, M. Alwan, S. Dalal, S. W. Kell, B. Turner, J. A. Stankovic, and R. Felder, "Behavioral Patterns of Older Adults in Assisted Living", IEEE transactions on information technology in biomedicine, Vol. 12, No. 3, May 2008.

[20] S. Ohta, H. Nakamoto, Y. Shinagawa, T. Kishimoto, "Home Telehealth: Connectng Care Within the Community.", Medical telematics, pp. 198209, 2006.

[21] K. N. Plataniotis and D. Hatzinakos, "Gaussian Mixtures and their Applications to Signal Processing", Department of Electrical and Computer Engineering, University of Toronto, March 10, 2000.

[22] J. Wyszynski, "Dichte-basierte Clusteringverfahren (G)DBSCAN", July 9th, 2007. 